FOUNDATIONS

ADVANCES
Keywords: Editorial; Acta Crystallographica Section $A$; modern crystallography

\section{Modern crystallography and its foundations}

\author{
Angela Altomare $^{\mathrm{a} *}$ and Simon J. L. Billinge $\mathrm{e}^{\mathrm{b}, \mathrm{c} *}$
}

\begin{abstract}
${ }^{\mathbf{a}}$ Institute of Crystallography of the National Research Council of Italy (IC-CNR), 70126 Bari, Italy, ${ }^{\mathbf{b}}$ Department of Applied Physics and Applied Mathematics, Columbia University, New York, NY 10027, USA, and ${ }^{\mathbf{c}}$ Condensed Matter Physics and Materials Science Department, Brookhaven National Laboratory, Upton, NY 11973, USA. *Correspondence e-mail: angela.altomare@ic.cnr.it, sb2896@columbia.edu
\end{abstract}

Acta Crystallographica Section A: Foundations and Advances (Acta A) has been the premier journal (see Billinge \& Miao, 2015) for foundational work in crystallography for the past 72 years (if we include the years when there was only one Acta Crystallographica). As we move into its 73rd year, we seek to maintain its prominence and impact, and this editorial is designed to share with you how you can help.

First, as described in our aims and scope (as captured at https://journals.iucr.org/a/), we remind our authors that, although 'crystal' is in the title of the journal, we accept articles about structural studies from a much broader array of materials, whether crystalline or not. For example, we welcome articles developing methodologies for single-particle imaging, studies of diffuse scattering and direct imaging of atoms. If you are reading this you already know this, but please share this with your colleagues!

We would also like to remind authors that, beyond the mathematical foundations of the subject, we also welcome articles that describe software and computational platforms that implement those algorithms. For example, analysis algorithms are moving in the direction of powerful but computationally demanding AI (artificial intelligence) and machine learning (ML), the data moving from tables to databases, and the analysis tools moving into the cloud (see, for example, Fig. 1), and we welcome articles in these areas.

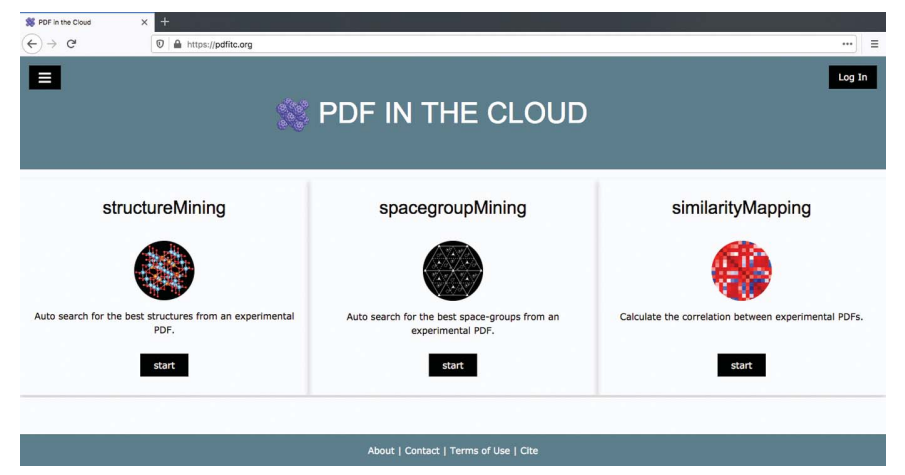

Figure 1

A cloud-based platform for atomic pair distribution analysis (see Yang et al., 2021).

Finally, we encourage authors to emphasize the crystallographic context of the work in their article. This is important for maintaining the relevancy, vibrancy and broader impact of the journal. In your view, who in the materials or structural communities will use it, and what will they use it for? We have found that, in these busy times, the more forward facing this information is the better. Can it be hinted at in the title? If not, it should appear in the abstract, or early in the introduction. It should also be captured in any short one- or twosentence synopsis about the work. In fact, such brief, public facing 'elevator pitches' are enormously valuable to us (and you!) to help promote the work and increase the readership. If you would like guidance in this regard, please feel welcome to contact our editorial team, who can make suggestions.

We thank you for your wonderful contributions and welcome even more in the upcoming years.

\section{References}

Billinge, S. J. L. \& Miao, J. (2015). Acta Cryst. A71, 1-2.

Yang, L., Culbertson, E. A., Thomas, N. K., Vuong, H. T., Kjær, E. T. S., Jensen, K. M. Ø., Tucker, M. G. \& Billinge, S. J. L. (2021). Acta Cryst. A77, 2-6. 